\title{
Long-term outcomes following minimal invasive versus conventional aortic valve replacement: a propensity match analysis
}

\author{
Gasparovic I, Artemiou P, Hudec V, Hulman M \\ Medical Faculty of the Slovak Health University, National Institute for Cardiovascular diseases, \\ Clinic of Cardiac Surgery, Bratislava, Slovakia. panayiotisartemiou@yahoo.com
}

\begin{abstract}
INTRODUCTION: Minimal invasive aortic valve replacement has become a routine procedure. In this study, we compared the outcomes between conventional and minimal invasive aortic valve replacement via the partial upper sternotomy that were performed in our Institution.

METHODS: The 5 year survival and postoperative outcomes of 34 patients that underwent isolated MIAVR between the years 2010-2013 were compared with the outcomes of 34 randomly selected patients that underwent conventional AVR, after propensity match analysis.

RESULTS: There was no difference between the two groups concerning the early and late postoperative outcomes. MIAVR patients had a longer mean cross-clamp time $(p=0.002)$ and longer cardiopulmonary bypass time $(p=0.0005)$ compared to the AVR patients. 5 year mortality and survival were $4.17 \%$ vs $16.67 \%(p=$ $0.20)$ and $95.8 \%$ vs $83.3 \%(p=0.37)$ in the MIAVR and AVR groups respectively.

CONCLUSION: This study showed a comparable 5 year survival and postoperative outcomes between the MIAVR and AVR groups. In our opinion, the minimal access aortic valve replacement can be performed safely with excellent long-term results in selected patients (Tab. 4, Fig. 1, Ref. 35). Text in PDF www.elis.sk.

KEY WORDS: minimal access aortic valve replacement, partial upper sternotomy.
\end{abstract}

\section{Introduction}

Aortic stenosis is the most commonly acquired heart valve lesion in the Western world. It is usually caused by degenerative changes with complex calcification of the native leaflets and aortic annulus. Aortic valve replacement (AVR) has been the gold standard for treatment of severe aortic stenosis for the last 40 years. It was first performed by Harken and Starr in 1960 (1) through a full median sternotomy and has been successfully performed in thousands of patients since then.

Interest in the minimally invasive aortic valve replacement (MIAVR) has increased after the adoption of transcatheter techniques to treat aortic stenosis and early feasibility studies on sutureless valve techniques (2-6). MIAVR was first performed by Cosgrove and Sabik in 1996 (7). MIAVR has been reported to offer several benefits over conventional full sternotomy procedures such as better cosmesis, reduced pain, reduced surgical trauma, decreased blood loss, earlier functional recovery and shorter hospital stay (8)

Medical Faculty of the Slovak Health University, National Institute for Cardiovascular diseases, Clinic of Cardiac Surgery, Bratislava, Slovakia.

Address for correspondence: P. Artemiou, Dr, National Institute of Cardiovascular Diseases, Pod Krasnou horkou 1, SK-831 01 Bratislava, Slovakia.

Phone: +421.917665774, Fax: +421.259320287
Various surgical approaches has been developed for MIAVR surgery. Currently, the most commonly performed MIAVR access is via a partial upper sternotomy that extends into the third of fourth intercostal space, referred to as a "J" or "L" sternotomy or an inverted "T" sternotomy $(9,10)$.

A minimal invasive aortic valve replacement has become routine in many institutions. In this study, we compare the outcomes between conventional and minimal invasive aortic valve replacement via the partial upper sternotomy that were performed in our Institution.

\section{Methods}

This is a retrospective observational study. In the study group, 34 patients were selected that underwent isolated MIAVR between February 2010 and January 2013. As the control group, 34 patients were randomly selected that underwent isolated conventional AVR during the same period. The patients were indicated for aortic valve replacement due to severe symptomatic aortic stenosis. After a propensity match analysis, two groups of 24 patients in each group were selected. Patients requiring concomitant procedures such as coronary artery bypass grafting, mitral or other valve surgery, replacement of the ascending aorta, or atrial fibrillation ablation were excluded. Patients undergoing aortic valve repair were also excluded. In the partial sternotomy group, 19 patients received a biologic and 5 patients received mechanical valves, where in the 
full sternotomy group 17 patients received biologic and 7 patients received mechanical valves.

\section{Patient selection}

The decision of whether patients underwent a MIAVR or a full sternotomy was predominantly made by the surgeon. Some surgeons exclusively used a MIAVR approach in all the patients. Other surgeons selectively applied MIAVR to those patients with a normal body-mass-index, a high risk of postoperative sternal wound infection, younger patients, or in those patients who explicitly requested a MIAVR approach. For MIAVR approach, we did not perform any additional preoperative investigations such as CT scans, MRI or transesophageal echocardiography.

\section{Outcome measures}

The primary outcome of this study was the 5 years survival. Secondary outcomes were intraoperative parameters like aortic cross-clamp and cardiopulmonary bypass times, presence of paravalvular leak at discharge, blood loss and transfusions, postoperative complications like respiratory (pneumonia, acute respiratory distress syndrome, pleural effusion) and renal complications, sternal wound infections, pacemaker implantation, postoperative atrial fibrillation, pain perception, mechanical ventilation time, rehabilitation process, postoperative length of stay and long-term complications like bleeding and thromboembolic complications, stroke and the presence of paravalvular leak

\section{Surgical technique and in-hospital treatment pathway}

The upper partial sternotomy without extension to the third or fourth intercostal space is the current standard approach for MIAVR in our institution. We utilized a $5 \mathrm{~cm}$ midline skin incision starting at the manubrium-sternal joint and performed a partial upper sternotomy without extension to the third or fourth intercostal space. The internal thoracic artery and vein remained intact. Direct antergrade aortic and right atrial appendage cannulations were performed. In some patients with short aortas, deeper location of the right atrial appendage or impaired working space femoral artery and vein cannulation through a 3-4 cm groin incision were performed. Myocardial protection consisted of antegrade and intermittent direct ostial administration of blood cardioplegia with mild hypothermia, or antegrade administration of crystalloid cardioplegia. The de-airing strategy included continuous $\mathrm{CO}_{2}$ flooding of the operative field, antegrade aortic root vent and transesophageal echocardiography confirmation of the de-airing efficiency in all cases. Standard techniques and instrumentation were used for incision and closure of the aorta, native valve and surrounding calcium removal followed by standard insertion of a biological or mechanical prosthesis.

Cardio-respiratory support, sedation and analgesia were administered as indicated in intensive care in a standard manner. Post-operative chest tubes were routinely removed 48 hours postoperatively and all patients received structured in-hospital and post-discharge rehabilitation.

Anticoagulation therapy with fenprocoumon (3M Health Care Ltd) ) was initiated and stabilized in hospital and continued for three months, with conversion to aspirin in the absence of persistent post-operative atrial fibrillation or mechanical valve implantation.

In the conventional full sternotomy AVR, a direct aortic and right appendage cannulations were performed, myocardial protection consisted of antegrade and intermittent direct ostial administration of blood cardioplegia with mild hypothermia, or antegrade administration of crystalloid cardioplegia. The de-airing strategy included continuous CO2 flooding of the operative field, antegrade aortic root and pulmonary artery vent and esophageal echocardiography confirmation of the de-airing efficiency in all the cases. Standard techniques were used to remove the native aortic valve and surrounding calcium, followed by standard insertion of a biological or mechanical prosthesis.

Transthoracic echocardiographic examinations were performed preoperatively, before discharge and at every follow-up meeting. Cardiac morphology and function as well as valve hemodynamics were assessed using standard measurements.

\section{Follow-up}

Follow-up was obtained by personal contact, or by phone with patients and family members, with supplemental information being supplied by family physicians and referring cardiologists. Valve related mortality and morbidity were evaluated according to the standard guidelines (11). The follow-up interval was 5 years and was completed in $90 \%$.

\section{Data analysis}

The study design was retrospective as the post-discharge data were collected retrospectively. Data are expressed as the mean \pm standard deviation. For the data analysis, a paired $t$ test was applied. For data correlation, the Pearson's respectively the Spearman's coefficients were applied. For a significant correlation was considered coefficient higher than 0.85 . A propensity-matched analysis was used to match the two study groups together. The Kaplan-Meier survival curve analysis was performed with the Greenwood formula of variance. In all the cases, $\mathrm{p}<0.05$ was considered significant. All analyses were performed using SPSS version 21.0 (IBM, USA)

\section{Results}

Each study group after a cross- matching consisted of 12 male (50\%) and 12 female (50\%) patients. The mean age in the MIAVR and the AVR groups were $60.2 \pm 15.8$ and $65 \pm 7.2(\mathrm{p}=0.19)$ respectively. The EUROSCORE II values were also $1.22 \pm 0.63$ and $1.36 \pm 1.11(\mathrm{p}=0.20)$ respectively. The preoperative patient characteristics are listed in Table 1.

Examination of the intraoperative variables revealed that MIAVR patients had the mean cross-clamp time of $63,4 \mathrm{~min} \pm 12.7$ compared to the AVR patients who had $50.3 \mathrm{~min} \pm 11.6$ (p = 0.002 ). The cardiopulmonary bypass time was $79.9 \mathrm{~min} \pm 14.9$ in the MIAVR group and $61.9 \mathrm{~min} \pm 13.7$ in the AVR group (p $=0.0005$ ). In the MIAVR group, 14 biologic and 5 mechanical valves were implanted, whereas in the AVR group were implanted 17 biological and 7 mechanical valves. The mean diameters of 
Tab. 1. Preoperative data.

\begin{tabular}{lccc}
\hline & MIAVR & AVR & $\mathrm{p}$ \\
\hline Age, years & $60.2 \pm 15.8$ & $65 \pm 7.2$ & 0.19 \\
Men & 12 & 12 & 1 \\
Female & 12 & 12 & 1 \\
Euroscore II & $1.22 \pm 0.63$ & $1.36 \pm 1.11$ & 0.20 \\
\hline
\end{tabular}

Tab. 2. Intraoperative data.

\begin{tabular}{lccc}
\hline Data & MIAVR & AVR & p \\
\hline Paravalvular leak & 2 & 2 & 1 \\
ACC (min) & $63.4 \pm 12.7$ & $50.3 \pm 11.6$ & 0.002 \\
CPB (min) & $79.9 \pm 14.9$ & $61.9 \pm 13.7$ & 0.0005 \\
Prosthetic valves & & & \\
Mechanical & 5 & 7 & \\
Biologic & 14 & 17 & \\
Valve size (mm) & & & \\
Mechanical & $22.4 \pm 1.74$ & $23.14 \pm 2.53$ & \\
Biologic & $22.16 \pm 1.76$ & $22.65 \pm 2.59$ & \\
\hline
\end{tabular}

ACC - Aortic cross clamp, CPB - Cardiopulmonary bypass

\section{Tab. 3. Early postoperative data}

\begin{tabular}{lccc}
\hline Data & MIAVR & AVR & p \\
\hline Blood loss (ml) & $340.20 \pm 200$ & $350 \pm 260.3$ & 0.73 \\
Transfusion (units) & $1.2 \pm 1.2$ & $1.3 \pm 1.5$ & 0.61 \\
Respiratory complications (pt) & 3 & 4 & 0.30 \\
Mechanical ventilation (hours) & $14.6 \pm 5.8$ & $15.3 \pm 7.9$ & 0.81 \\
Early rehabilitation (pt) & 18 & 23 & 0.08 \\
Pain* & $4.13 \pm 1.30$ & $4.03 \pm 1.09$ & 0.93 \\
Atrial fibrillation (pt) & 9 & 12 & 0.39 \\
Renal dialysis (pt) & 0 & 0 & 1 \\
Sternal wound infection (pt) & 3 & 1 & 0.31 \\
ICU stay (days) & $4 \pm 2.6$ & $4.8 \pm 3.1$ & 0.32 \\
Hospitalization (days) & $13.3 \pm 6.1$ & $12.8 \pm 8.3$ & 0.93 \\
\hline *
\end{tabular}

* according to the pain scale, ICU - Intensive care unit, pt - patient

Tab. 4. Long-term data.

\begin{tabular}{lccc}
\hline Data & MIAVR & AVR & p \\
\hline Mortality (pt) & 1 & 4 & 0.20 \\
Thrombosis (bleeding (pt) & 0 & 0 & 1 \\
Paravalvular leak (pt) & 1 & 0 & 0.33 \\
Cerebrovascular stroke (pt) & 0 & 0 & 1 \\
Pacemaker implantation (pt) & 1 & 1 & 0.95 \\
\hline
\end{tabular}

the biological and mechanical valves in the MIAVR group were $22.16 \pm 1.76$ and $22.4 \pm 1.74$ respectively. In the AVR group, the mean diameters of the biologic and mechanical valves were 22.65 \pm 2.59 and $23.14 \pm 2.53$ respectively. Two patients (8.3\%) in the MIAVR group had a mild paravalvular leak and two patients (8.3 \%) in the AVR group had trivial and moderate paravalvular leak, respectively. The intraoperative characteristics are listed in Table 2.

Examination of the early postoperative outcomes revealed that the mean blood loss was $340.2 \mathrm{ml} \pm 200$ in the MIAVR group and $350 \pm 260.3$ in the AVR group ( $\mathrm{p}=0.73)$ and the mean use of red pack cells was $1.2 \pm 1.2$ and $1.3 \pm 1.5$ units in the MIAVR and AVR groups respectively ( $\mathrm{p}=0.61$ ). The mean time in the mechanical ventilator was $14.6 \pm 5.8$ and $15.3 \pm 7.9$ hours in the
MIAVR and AVR groups, respectively $(\mathrm{p}=0.81)$. Three patients (12.5 \%) in the MIAVR group and 1 (4.17 \%) patient in the AVR had respiratory complications ( $p=0.3)$. Nine patients $(37.5 \%)$ in the MIAVR group and 12 patients (50\%) in the AVR group had paroxysmal postoperative atrial fibrillation $(\mathrm{p}=0.39)$. No patient in both group had perioperative renal dialysis due to acute renal failure and 3 patients (12.5\%) in the MIAVR group and 1 (4.17 $\%)$ patient in the AVR group had sternal wound infection ( $\mathrm{p}=$ 0.31). Eighteen patients (75 \%) in the MIAVR group and 23 (95.8 $\%)$ patients in the AVR group underwent a normal rehabilitation process $(p=0.08)$. Moreover, the postoperative pain perception in both groups was not statistically significant ( $p=0.93$ ). The mean intensive care unit stay was $4 \pm 2.6$ and $4.8 \pm 3.1$ days in the MIAVR and AVR groups, respectively $(p=0.32)$. Finally, the mean hospital stay was $13.3 \pm 6.1$ and $12.8 \pm 8.3$ days in the MIAVR and AVR groups, respectively ( $\mathrm{p}=0.93$ ). The early postoperative characteristics are listed in the Table 3.

Examination of the long-term postoperative outcomes revealed that no patients from both groups had any thrombotic, bleeding or cerebrovascular complications. One patient $(4.17 \%)$ in the MIAVR and 1 patient ( $4.17 \%)$ in the AVR group had a pacemaker implantation following the procedure $(\mathrm{p}=0.95)$. In the long-term follow-up, only one patient (4.17\%) from the MIAVR had a mild paravalvular leak. In the AVR group, no paravalvular leak was observed $(\mathrm{p}=0.33)$.

Finally, in the long-term, no difference in the mortality was observed (one patient (4.17\%) in the MIAVR group, 4 patients ( $16.67 \%$ ) in the AVR group, $\mathrm{p}=0.20$ ). The long-term postoperative characteristics are listed in the Table 4.

There was no difference in the 5 year long-term survival between the two groups (in MIAVR group $95.8 \%$, in AVR group $83.3 \%(\mathrm{p}=0.37)$. The survival is shown in the Kaplan-Meier survival curve in the Figure 1.

In the MIAVR group, there was a strong correlation between the aortic cross clamp and the cardiopulmonary by-pass times and the total hospitalization time $(r=0.91, p=0.000)$. Also, the same strong correlation between the aortic cross clamp and cardiopulmonary by-pass times was seen in the AVR group ( $\mathrm{r}=0.91$, $p=0.000$ ). That means that in both groups, the longer the aortic

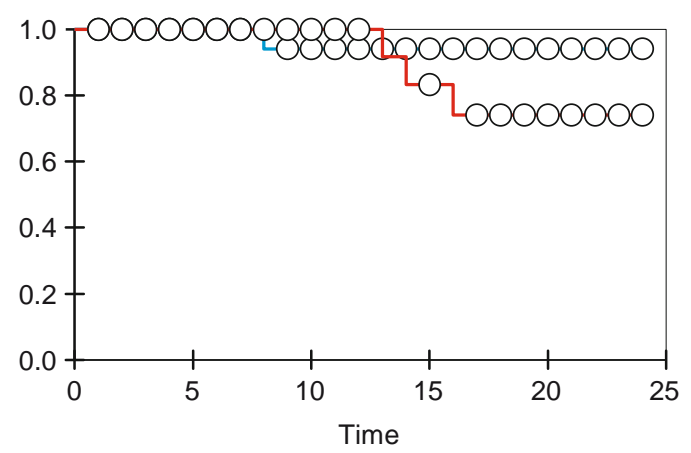

Fig. 1. Kaplan-Meier survival curve. Blue line - MIAVR, red line AVR p $=0.37$. 
cross clamp and cardiopulmonary by-pass times, the longer the hospitalization of the patient. In the AVR group, there was a weak correlation between the respiratory complications and the transfusion of red pack cells $(r=0.61, p=0.00173)$ and that means the increased transfusion of red pack cells increases the risk for pulmonary complications.

\section{Discussion}

The excellent outcomes of the current conventional surgical techniques for valvular disease set high standards for the implementation and development of new approaches and strategies, especially in view of an aging population with increased comorbidities, operative risks and quality of life expectations (12). A number of previous publications have shown that MIAVR is superior to a conventional AVR due to shorter hospitalization stay, reduced postoperative ventilation time, less blood loss and lower transfusion rates (13-16). Although some studies have found contrary results with no obvious benefit for a minimally invasive approach $(17,18)$, a meta-analysis has confirmed the above mentioned advantages (8). In our study, MIAVR had no obvious benefit comparing to the conventional AVR.

Although MIAVR has several benefits, it is also associated with a longer aortic cross-clamp, CPB and surgical times (8) as also shown in our study probably because of an increased technical difficulty posed by the reduced surgical field. The longer myocardial ischemic and CPB times that have been reported in MIAVR patients have not been shown to increase the rate of related adverse effects such as, myocardial infarction, intra-aortic balloon pump use or low cardiac output syndrome in MIAVR patients $(8,14$, $16,19,20)$. In our study, the longer aortic cross-clamp and CPB times observed in the MIAVR group did not have any impact on the incidence of postoperative complications such as the renal and respiratory complications, presence of postoperative atrial fibrillation, sternal wound infection and pain perception.

MIAVR might offer additional advantages to the sutureless valve technologies that are perceived to be an alternative treatment for high risk patients with aortic stenosis $(3,21)$. Comparison with STS data showed $60 \%$ decrease in the operative time, which might reduce the effects on myocardial ischemia and hypoxia (16). The long-term results of these devices remain unknown. In our study, sutureless valves were not used.

In contrast to our study, where we did not observe any difference in the various postoperative complications, the hospital stay, the ventilation time, the blood loss and red blood cells transfusions, Glauber et al (19) demonstrated a lower incidence of postoperative atrial fibrillation and blood transfusion, as well as shorter ventilation times and hospital stay in MIAVR patients.

Reduction of postoperative bleeding and the need for blood transfusions are the two most common mentioned benefits of MIAVR. On the contrary, in our study we did not observe any difference. Different authors $(8,14,22-24)$ claim this benefit of MIAVR over the conventional AVR.

There are numerous reports of a shorter hospitalization in MIAVR patients in literature $(8,14,19)$. In our study, we did not observe any difference in the hospitalization time between the MIAVR and AVR groups.

Large comparative studies $(24,25)$ failed to reveal superiority of MIAVR in sternal wound infection prevention. Also, in our study, the rate of sternal wound infection was similar between the two groups.

We did not observe any conversion to full sternotomy in our study. Published data on conversion show it to be in the range of $1 \%$ to $3 \%$ (15). We believe that a detailed preoperative planning and a relatively good clinical experience may have contributed to the ability to avoid a full sternotomy in MIAVR patients.

An important issue considering the particularly important advantage of MIAVR is the potential for faster recovery. Better stability of the sternum and thorax leads to improvement of the patients respiratory function and earlier mobilization translates into a shorter mechanical ventilation support, shorter intensive care unit and overall hospital stay, and shorter time required for rehabilitation. In our study, we did not observe any difference between the MIAVR and AVR groups concerning the above mentioned parameters. On the contrary, other authors demonstrated a reduction in time spent in hospital for MIAVR patients $(8,24,26)$. A meta-analysis of available randomized control trials has been conducted with the conclusion that the length of intensive care unit stay was significantly shorter in favor of the mini-sternotomy group (27). In the Slovak medical system, the impact on length of hospital stay,may be explained by the vagaries of reimbursement in the hospital system, complicating comparisons of hospital stays to those from other countries.

Among other aspects that can contribute to faster recovery, the postoperative pain is of great importance. Indeed its intensity is sometimes problematic to estimate, because of the individual patient's threshold. Logically, the minimally invasive approach should cause less pain and discomfort postoperatively. In this study, the postoperative pain intensity was measured with the use of the pain scale with reference numbers from 1 to 10 and the patients in both study groups had the same pain perception postoperatively. On the contrary, Yamada et al (28) in a retrospective study demonstrated that mini-invasive patients had earlier recovery and improved quality of life with diminished pain medication administration compared to the conventional AVR population. Other authors $(29,30)$ demonstrated similar results. Moreover, in our study, patients from the both study groups had similar rehabilitation processes.

In this study, the 5 year mortality in the MIAVR group was 4.17 $\%$ and in the AVR group $16.67 \%$, but did not reached a statistical significance. Merck et al (20) in a propensity matched analysis demonstrated also a significantly reduced long term mortality in the MIAVR group. Similarly, Mihaljevic et al (15) noted a reduced mortality for patients undergoing MIAVR.

Moreover, this study demonstrated that MIAVR had comparable outcomes with regard to long-term survival analyzed at 5 years (in the MIAVR group $95.8 \%$, in the AVR group $83.3 \%$ ). Similar results with comparable outcomes were presented by Attia RQ et al (31) with the 5 year survival in the MIAVR group at $87.5 \%$ and in the AVR group $85.5 \%$. Merck et al (20) showed an 
absolute increase in postoperative survival of $7.5 \%$ and $4.9 \%$ at 5 and 8 years respectively, when compared to conventional AVR surgery. Glauber et al (19) demonstrated an excellent survival in MIAVR patients three years postoperatively (96\% vs $88 \%$ for conventional AVR group) but this difference did not reach statistical significance. Comparable survival at 5 years of $83.8 \pm 1.1 \%$ as in our study was presented by Lehmann et al (32).

The long-term functional outcomes of MIAVR, including valve durability, low incidence of stroke and presence of paravalvular leak as shown in this and other studies $(33,34)$ are excellent and comparable with the conventional AVR outcomes.

Minimal aortic valve replacement is often combined with the implantation of sutureless valves. The use of sutureless valves simplifies the operation and makes it less risky for the patient (35). However, in our study group, we did not use sutureless valves. Minimal invasive AVR and implantation of sutureless valves is a win-win situation, because it combines the benefits of both. However, the use of stented bioprosthetic and mechanical valves, as in our study, offers a safe and a durable result.

This is a retrospective propensity matched study that analyzed the 5 year survival rate and secondary outcomes between the minimal invasive and conventional AVR. The study weakness is the low number of patients that were enrolled.

In conclusion, this study showed comparable 5 year survival and postoperative outcomes between the MIAVR and AVR groups. In our opinion, the minimal access aortic valve replacement can be performed safely with excellent long-term results in selected patients. The lack of clear benefit serves as a call for a large randomized trial to conclusively define clinical differences between full sternotomy and less invasive approaches.

\section{References}

1. Harken DE, Soroff HS, Taylor WH. Aortic valve replacement. In: Prosthetic valves in cardiac surgery. Merendino KA(Ed). Thomas, Springfield, IL 1961: 508-521.

2. Smith CR, Leon MB, Mack MJ et al. Trancatheter versus surgical aortic valve replacement in high-risk patients. N Engl J Med 2011: 364: 2187-2198.

3. Kocher AA, Laufer G, Haverich A et al. One year outcomes of the surgical tratment of aortic stenosis with a next generation surgical aortic valve (TRITON) trial: a prospective multicenter study of rapid deployment with the EDWARDS INTUITY valve system. J Thorac Cardiovasc Surg 2013: 145: 110-116.

4. Kodali SK, Williams MR, Smith CR et al. Two-year outcomes after trancatheter or surgical aortic valve replacement. N Engl J Med 2012: 366: 1686-1695.

5. Santarpino G, Pfeifer S, Schmidt J, Concistre G, Fischlein T. Suturless aortic valve replacement: first-year single center experience. Ann Thorac Surg 2012: 94: 504-509.

6. Santarpino G, Pfeifer S, Jessl J et al. Suturless replacement versus transcatheter valve implantation in aortic valve stenosis: a propensitymatched analysis of 2 strategies in high risk patients. J Thorac Cardiovasc Surg 2014: 147: 561-567.
7. Cosgrove DM 3rd, Sabik JF. Minimally invasive approach for aortic valve operations. Ann Thorac Surg 1996: 62: 596-597.

8. Murtuza B, Pepper JR, Stanbridge RD et al. Minimal access aortic valve replacent: is it worth it? Ann Thorac Surg 2008: 85: 1121-1131.

9. Svensson LG, D’Agostino RS. "J" incision minimal -access valve operations. Ann Thorac Surg 1998: 66: 1110-1112.

10. Farhat F, Lu Z, Lefevre $\mathbf{M}$ et al. Prospective comparison between total sternotomy and ministernotomy for aortic valve replacement. J Card Surg 2003: 18: 396-401, discussion 402-403.

11. Akins CW, Miller DC, Turina MI et al. Guidelines for reporting mortality and morbidity after cardiac valves interventions. Eur J Cardiothorac 2008: 33: 523-528.

12. Phan K, Xie A, Di Eusanio M et al. A meta-analysis of minimally invasive versus conventional sternotomy for aortic valve replacement, Ann Thorac Surg 2014: 98: 1499-1511.

13. Shehada SE, Ozturk O, Wottke M, Lange R. Propensity score analysis of outcomes following minimal access versus conventional aortic valve replacement. Eur J Cardiothorac Surg 2016: 49(2): 464-470.

14. Bonacci M, Prifti E, Giunti G et al. Does ministernotomy improve postoperative outcome in aortic valve operation? A prospective randomized study. Ann Thorac Surg 2002: 73: 460-465.

15. Mihalijevic T, Cohn LH, Unic D et al. One thousand minimally invasive valve operations: early and late results. Ann Surg 2004: 240: 529-534.

16. Tabata M, Umakanthan R, Cohn LH et al. Early and late outcomes of 1000 minimally invasive aortic valve operations, Eur J Cardiothorac Surg 2008: 33: 537-541.

17. Christiansen S, Stypmann J, Tjan TD et al. Minimally-invasive versus conventional aortic valve replacement-perioperative course and mid-term results. Eur J Cardiothorac Surg 1999: 16: 647-652.

18. Detter C, Deuse T, Boehm DH et al. Midterm results and quality of life minimally invasive vs conventional aortic valve replacement. Thorac Cardiovasc Surg 2002: 50: 337-341.

19. Glauber M, Miceli A, Gilmanov D et al. Right anterior minithoracotomy versus conventional aortic valve replacement: a propensity score matched study. J Thorac Cardiovasc Surg 2013: 145: 1222-1226.

20. Merck DR, Lehmann S, Holzhey DM et al. Minimal invasive aortic valve replacement surgery is associated with improved survival: a propensity- matched comparison. Eur J Cardiothorac Surg 2015: 47(1): 11-17.

21. Fischlein T. Pfeiffer S, Poliari F, Sirch J, Vogt F, Santarpino G. Sutureless valve implantation via mini J-sternotomy: a single center experience with 2 years mean follow-up. Thorac Cardiovasc Surg 2015: 63(6) 467-471.

22. Dogan S, Dzemali O, Wimmer-Greinecker G et al. Minimally invasive versus conventional aortic valve replacement: a prospective randomized trial. J Heart Valve Dis 2003: 12: 76-80.

23. Gilmanov D, Bevilacqua S, Murzi M, et ai. Minimally invasive and conventional aortic valve replacement : a propensity score analysis. Ann Thorac Surg 2013: 96: 837-843.

24. Browm ML, McKellar SH, Sundt TM, Schaff HV. Ministernotomy versus conventional sternotomy for aortic valve replacement: a systematic review and meta-analysis. J Thorac Cardiovasc Surg 2009: 137: 670-679.

25. Johnston DR, Atik FA, Rajeswaran J et al. Outcomes of less invasive J-incision approach to aortic valve surgery. J Thorac Cardiovasc Surg 2012: 144: 852-858 . 


\section{9-484}

26. Sharony R, Grossi EA, Saunders PC et al. Minimally invasive aortic valve surgery in the elderly: a case-control study. Circulation 2003: 108: Suppl 1: 1143-1147.

27. Khosbin E, Prayaka S, Kinsella J, Sutherland FW. Mini-sternotomy for aortic valve replacement reduces the length of stay in the cardiac intensive care unit.: meta-analysis of randomized controlled trials BMJ Open 2011: 1(2): e000266.

28. Yamada T, Ochiai R, Takeda J, Shin H, Yozu R. Comparison of early postoperative quality of life in minimally invasive versus conventional valve surgery. J Anest 2003: 17: 171-176.

29. Candele SI, Herijgers P, Demeyere R, Flameng W, Evers G. Chest pain after partial upper sternotomyfor aortic valve surgery. Acta Cardiol 2003: 58: 17-21.

30. Machler HE, Bergmann P. Minimally invasive versus conventional aortic valve operations: a prospective study in 120 patients. Ann Thorac Surg 1999: 67: 781-785.
31. Attia RQ, Hickey GL, Grant SW et al. Minimally invasive versus conventional aortic valve replacement. A propensity-matched study from the UK national data. Innovations 2016: 11: 15-23.

32. Lehmann S, Merk DR, Etz CD et al. Minimally invasive aortic valve replacement: the Leipzig experience. Ann Cardiothorac Surg 2015: 4(1): 49-56.

33. Salenger R, Gammie JS, Collins J. Minimally invasive aortic valve replacement. J Card Surg 2016: 31: 38-50.

34. Kaczmarczyk M, Szalanski P, Zembala M et al. Minimally invasive aortic valve replacement-pros and cons of keyhole aortic surgery. Kardiochirurgia I Torakochirurgia Polska 2015: 12(2): 103-110.

35. Gilmanov D, Miceli A, Belivacqua S et al. Sutureless implantation of the Perceval S, aortic valve prosthesis through right anterior minithoracotomy. Ann Thorac Surg 2013: 96: 2101-2108.

Received March 29, 2017. Accepted April 20, 2017. 